\title{
Dynamic modelling of a Condenser with the ThermoSysPro Library
}

\author{
Baligh El Hefni Daniel Bouskela \\ EDF R\&D \\ 6 quai Watier, 78401 Chatou Cedex, France \\ baligh.el-hefni@edf.fr daniel.bouskela@edf.fr
}

\begin{abstract}
The condenser is an important device for the operation of power plants in particular for pressurized water reactors. Undesirable transients may lead to the automatic shutdown of the power plant.

To simulate the complex dynamic physical behaviour of the condenser, a dynamic model has been developed using Modelica. The component model is meant to be used for power plant modeling and simulation with the ThermoSysPro library developed by EDF and released under open source license.

The transient most unfavorable to keeping the integrity of the condenser vacuum is the loss of the cold source pumps followed by a turbine trip and a missed islanding (house load operation).

The objective is to study the evolution of the pressure of the condenser for this kind of transients. During the transiernt, the condenser pressure should be always less than 5e4 Pa.

The present paper describes in detail the condenser model: hypothesis, governing equations, correlations and the test-case (structure of the test model, the parameterization data and the results of simulation).
\end{abstract}

Keywords: Modelica; thermal-hydraulics; heat exchange; condenser; dynamic modeling; inverse problems; ThermoSysPro

\section{Introduction}

Modeling and simulation activities play a key role in the design phase and performance optimization of complex energy processes. It is also expected that they will play a significant role in the future for power plant maintenance and operation.

The potential of Modelica as a mean to efficiently describe thermodynamic models has been recognized for quite a while [1, 2], and has led to the initiative of developing an EDF library for power plant modeling within the ITEA 2 EUROSYSLIB project.
This library, called ThermoSysPro, aims at providing the most frequently used models of components for the 0D-1D static and dynamic modeling of thermodynamic systems, mainly for power plants, but also for other types of energy systems such as industrial processes, energy conversion systems, buildings etc. It involves disciplines such as thermal-hydraulics, combustion, neutronics and solar radiation (see instance [1 to 8].

The ambition of the library is to cover all the phases of the plant lifecycle, from basic design to plant operation. This includes for instance system design, verification and validation of the instrumentation and control system, system diagnostics and plant monitoring. To that end, the library will be linked in the future to systems engineering via the modeling of systems properties (or requirements), and to the measurements made on the real process via state estimation techniques.

The present paper focuses on the dynamic modeling of the condenser: hypothesis, governing equations, correlations and the test-case (structure of the model, the parameterization data and the results of simulation).

\section{Model of the condenser}

\subsection{General presentation of the condenser}

The condenser is a two-phase shell-and-tube heat exchanger. The feedwater flows inside the tube bundle, while the steam and condensate flows outside of those tubes located inside the cavity. In the condenser, there are two zones: the desuperheating zone and the condensation zone.

The condensate of water heaters located upstream is injected into the condenser. During the injection, part of the condensate may vaporize due to the pressure drop. This phenomenon is known as flash. 


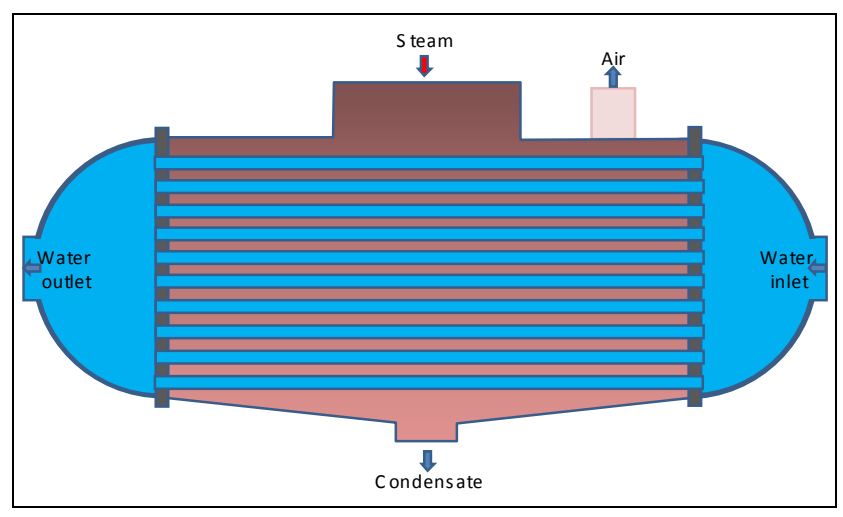

Figure 1: fluid flow inside an condenser

\subsection{Description of the condenser model}

The DynamicCondenser model represents the dynamics of the thermo-hydraulic phenomena of the hot fluid inside the cavity and of the cooling fluid which flows through the tube bundle. In particular, the model features the thermal exchanges between the fluid in the cavity and the cooling fluid flowing through the tube bundle.

The condenser is considered as a vertical or horizontal cylindrical cavity (as schematized in Figure 1), containing a tube bundle with the feedwater inlet and outlet located on the end.

\subsection{Components of the condenser}

The model is divided into sub-models of three different types which are connected together to make the full model (see Figure 2):

- One DynamicOnePhaseFlowPipe model,

- One HeatExchangerWall model,

- One TwoPhaseCavityOnePipe model.

By reassembling the sub-models, any other configuration of the condenser can be modelled.

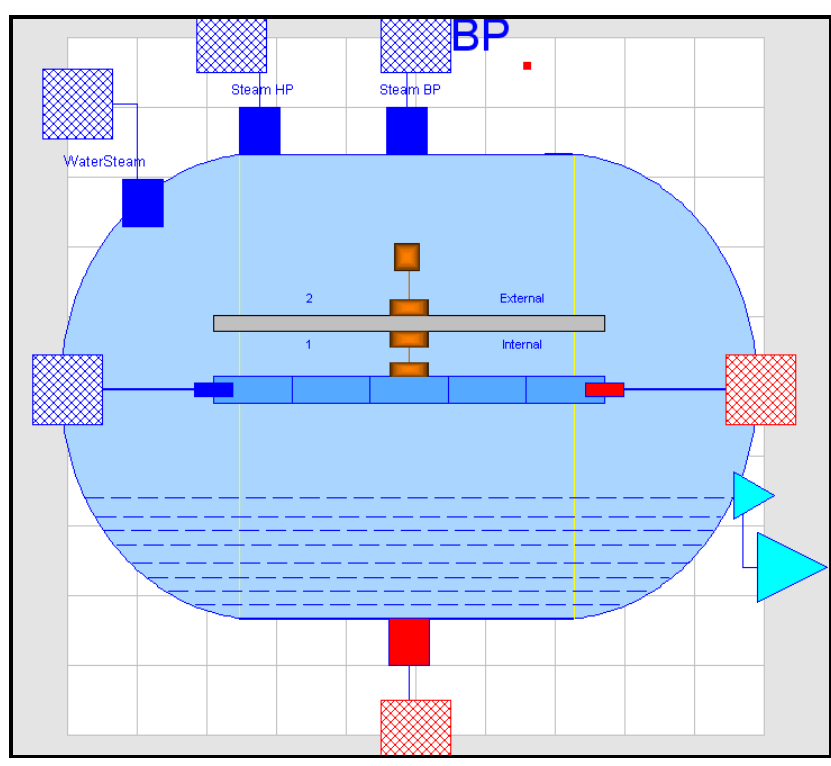

Figure 2: icon representation of the component model

The description of each sub-model is given in the following section. Each sub-model in the model can be recognized by looking at its icon (see Figures 3, 4 and 6).

\section{Physics of the condenser}

\subsection{DynamicOnePhaseFlowPipe model}

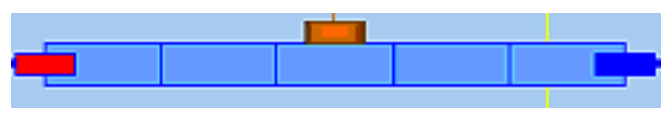

Figure 3: pipe model icon

The model of the fluid flow in a cylindrical conduit is based on the dynamic mass, energy, and momentum balance equations, which are originally given as 1-D partial differential equations. The original distributed-parameter model is first discretised by using the finite-volume method. The model is formulated in order to correctly handle possible flow reversal conditions. 


\section{$\underline{\text { Assumptions }}$}

- Homogeneous fluid in each mesh cell (same velocity for the liquid and steam phases);

- 1-D modelling (using the finite-volume method);

- The accumulation is considered in each mesh cell;

- The inertia of the fluid is taken into account;

- The phenomenon of longitudinal heat conduction in the metal wall and in the fluid is neglected;

- The thermo-physical properties are calculated on the basis of the average pressure and enthalpy in each mesh cell.

\section{Mass balance equation}

The mass balance equation in each cell is given by:

$$
A \cdot \frac{d \rho_{i}}{d t} \cdot \Delta x=\dot{m}_{i-1: i}-\dot{m}_{i: i+1}
$$

Taking the pressure and the specific enthalpy as state variables yields:

$A \cdot\left[\left(\frac{\partial \rho_{i}}{\partial P_{i}}\right)_{h} \cdot \frac{d P_{i}}{d t}+\left(\frac{\partial \rho_{i}}{\partial h_{i}}\right)_{P} \cdot \frac{d h_{i}}{d t}\right] \cdot \Delta x=\dot{m}_{i-1: i}-\dot{m}_{i: i+1}$

\section{Energy balance equation}

The energy balance equation in each cell is given by: $A \cdot \frac{d\left(\rho_{i} \cdot u_{i}\right)}{d t} \cdot \Delta x=\dot{m}_{i-1: i} \cdot h_{i-1: i}-\dot{m}_{i: i+1} \cdot h_{i: i+1}+\Delta W_{i}$

with the specific internal energy given by:

$$
u_{i}=h_{i}-\frac{P_{i}}{\rho_{i}}
$$

Taking the pressure and the specific enthalpy as state variables yields:

$$
\begin{gathered}
A .\left(\left(h_{i} \cdot \frac{\partial \rho_{i}}{\partial P_{i}}-1\right) \cdot \frac{d P_{i}}{d t}+\left(h_{i} \cdot \frac{\partial \rho_{i}}{\partial h_{i}}+\rho_{i}\right) \cdot \frac{d h_{i}}{d t}\right) \cdot \Delta x \\
\dot{m}_{i-1: i} \cdot h_{i-1: i}-\dot{m}_{i: i+1} \cdot h_{i: i+1}+\Delta W_{i}
\end{gathered}
$$

$h_{i: i+1}$ is the specific enthalpy of the mass flow $\dot{m}_{i: i+1}$ crossing the boundary between the cells $i$ and $i+1 . h_{i: i+1}$ is related to the state variables $h_{i}$ and $h_{i+1}$ by:

$$
h_{i: i+1}=\hat{s}\left(P_{e}\right) \cdot h_{i}+\hat{s}\left(-P_{e}\right) \cdot h_{i+1}
$$

where $P_{e}$ is the Peclet number and

$$
\widehat{s}(x)=\frac{1}{1+e^{-\frac{x}{2}}} \text { (see e.g. [10]). }
$$

When neglecting diffusion, the Peclet number is infinite, and

$$
h_{i: i+1}=s\left(\dot{m}_{i: i+1}\right) \cdot h_{i}+s\left(-\dot{m}_{i: i+1}\right) \cdot h_{i+1}
$$

Where $s$ is the step function:

$$
s(x)=\left\{\begin{array}{lll}
1 & \text { if } & x>0 \\
0 & \text { if } & x<0
\end{array}\right.
$$

This simplification is known as the upwind scheme.

\section{$\underline{\text { Momentum balance equation }}$}

The momentum balance equation in each cell is given by:

$$
\begin{aligned}
1 / A \cdot & \frac{d \dot{m}_{i: i+1}}{d t} \cdot \Delta x= \\
& P_{i}-P_{i+1}-(\Delta P)_{i: i+1}^{a}-(\Delta P)_{i: i+1}^{f}-(\Delta P)_{i: i+1}^{g}
\end{aligned}
$$

with respectively the acceleration, friction and gravity pressure losses given by:

$$
\begin{aligned}
& (\Delta P)_{i: i+1}^{a}=\frac{1}{A^{2}} \cdot \dot{m}_{i: i+1} \cdot\left|\dot{m}_{i: i+1}\right| \cdot\left(\frac{1}{\rho_{i+1}}-\frac{1}{\rho_{i}}\right) \\
& (\Delta P)_{i: i+1}^{f}=\zeta \cdot \frac{\Lambda_{i} \cdot \Delta x_{h}}{2 \cdot D \cdot A^{2} \cdot \rho_{i}} \cdot \dot{m}_{i: i+1} \cdot\left|\dot{m}_{i: i+1}\right| \\
& (\Delta P)_{i: i+1}^{g}=\rho_{i: i+1} \cdot g \cdot\left(z_{i+1}-z_{i}\right)
\end{aligned}
$$

By default, the flow is considered turbulent (Reynolds number Re > 2300).

The Colebrook correlation is used to compute $\Lambda_{i}$.

\section{Convective heat transfer within the tubes}

The heat exchanged between the fluid and the wall is:

$$
\Delta W(i)=h_{c}(i) \cdot \Delta S_{2} \cdot\left(T_{w 2}(i)-T(i)\right)
$$

\section{Convection heat transfer coefficient}

The convection heat transfer coefficient $h_{c}$ between the fluid and the wall is computed using the DittusBoelter correlation. 


\subsection{HeatExchangerWall model}

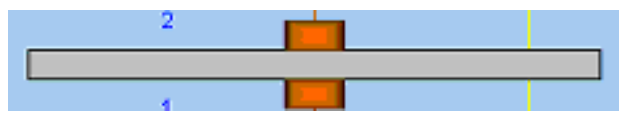

Figure 4: wall model icon

The wall model describes the conductive heat flow through the wall of the tube bundle. The flow is positive when entering the tubes (going from side 2 to side 1 of the wall).

$$
\begin{aligned}
& \Delta W_{1}(i)=\frac{2 \cdot \pi \cdot \lambda \cdot \Delta x(i) \cdot \text { ntubes } \cdot\left(T_{w}(i)-T_{w 1}(i)\right)}{\ln ((e+D) / D)} \\
& \Delta W_{2}(i)=\frac{2 \cdot \pi \cdot \lambda \cdot \Delta x(i) \cdot n t u b e s \cdot\left(T_{w 2}(i)-T_{w}(i)\right)}{\ln ((2 \cdot e+D) /(e+D))} \\
& \Delta M_{w} \cdot c_{p w} \cdot \frac{d T_{w}}{d t}=\Delta W_{2}(i)-\Delta W_{1}(i)
\end{aligned}
$$

\subsection{TwoPhaseCavityOnePipe model}

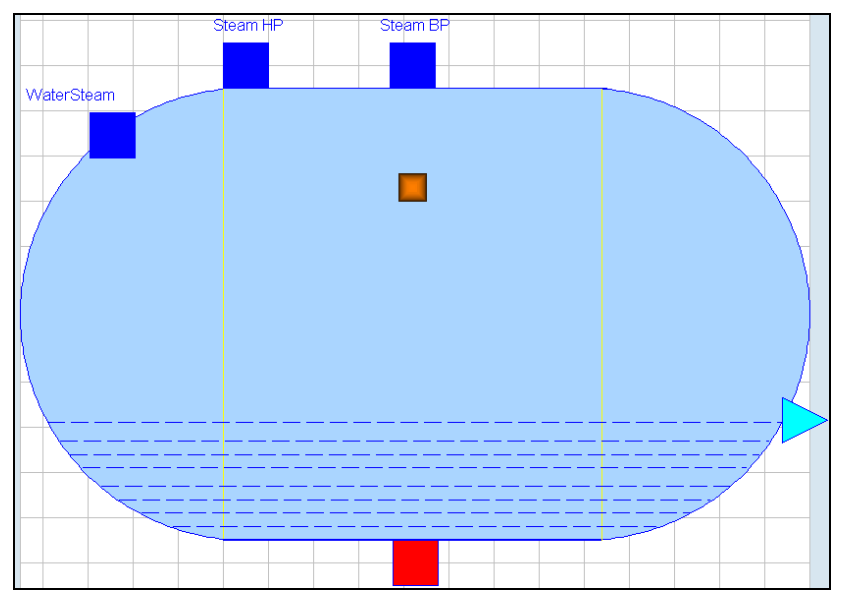

Figure 5: two-phase cavity model icon

The cavity is modelled as a non-adiabatic two-phase volume, with vertical or horizontal cylindrical geometry. The physical model is based on a nonequilibrium, two-phase formulation of the fluid balance equations with a control volume approach. The two phases are supposed to be isobaric and will be referred to as liquid zone and steam zone, respectively.

The model features the condensation flow of the steam phase into the liquid phase.

In most operating conditions, the liquid and steam phases in cavity are not necessarily in thermal equilibrium. The reasons are:
- The vapour may enter the cavity in a superheated state (the vapour temperature is then higher than the saturation temperature).

- The liquid may be subcooled by the incoming drain.

\section{$\underline{\text { Assumptions }}$}

- Accumulation of mass and energy is considered. Heat exchange between the liquid and steam phases is considered.

- Heat exchange between the liquid or steam phases and the wall is considered.

- Heat exchange between the condenser and the external medium (ambient) is considered.

- Pressure losses are not taken into account in the cavity.

- The liquid and steam phases are not necessarily in thermal equilibrium.

- The liquid and steam phases are assumed to be permanently in pressure equilibrium.

\section{State variables}

The state variables of the system are:

- the mean pressure in the cavity,

- the specific enthalpy of the liquid phase,

- the specific enthalpy of the steam phase,

- the temperature of the wall,

- the volume of the liquid phase.

The volume of the steam phase is bound to the volume of the liquid phase by the following equation:

$$
V_{l}+V_{v}=V
$$

\section{Mass balance equation in each phase}

$$
\begin{aligned}
& \frac{d\left(\rho_{l} \cdot V_{l}\right)}{d t}=-\dot{m}_{l}^{o}+\left(1-x_{m v}\right) \cdot \dot{m}_{d r a i n}^{e}+\dot{m}_{c o n d} \\
& \frac{d\left(\rho_{v} \cdot V_{v}\right)}{d t}=\dot{m}_{v}^{e}+x_{m v} \cdot \dot{m}_{d r a i n}^{e}-\dot{m}_{c o n d}
\end{aligned}
$$

where $\dot{m}_{v}^{e}$ is the mass flow of incoming vapor, $\dot{m}_{\text {drain }}^{e}$ is the mass flow of the incoming condensate of the some water heaters, $\dot{m}_{l}^{o}$ is the mass flow of outgoing condensate and $\dot{m}_{\text {cond }}$ is the condensation flow inside the cavity. 
Condensation mass flow rate inside the cavity

The evolution of the pressure in the condenser depends strongly upon the mass flow rate of condensation.

The classical equation used to calculate the condensation mass flow rate given in [8] is not correct to simulate the scenario "loss of the cold source pumps followed by a turbine trip and a missed islanding". The following new equation gives correct results:

$$
\begin{aligned}
& \dot{m}_{\text {cond_1 }}=\frac{W_{1 t}+W_{2 t}+W_{v l}+W_{v p}}{h_{v}^{\text {sat }}-h_{l}^{s a t}} \\
& \dot{m}_{\text {cond_2 }}=\left(1-x_{e}^{v}\right) \cdot \dot{m}_{v}^{e} \\
& \dot{m}_{\text {cond }}=\dot{m}_{\text {cond_1 }}+\dot{m}_{\text {cond_2 }}
\end{aligned}
$$

where $\dot{m}_{\text {cond } 11}$ is the mass flow rate of the steam condensed in the cavity and $\dot{m}_{\text {cond } 22}$ is the mass flow rate of the water contained in the wet steam at the cavity inlet.

\section{Energy balance equation in each phase}

The general form of the energy balance equation is given by:

$$
\frac{d(\rho \cdot V \cdot u)}{d t}=\sum_{e} \dot{m}_{e} \cdot h_{e}+\sum_{o} \dot{m}_{o} \cdot h_{o}+\sum W
$$

Taking the pressure and the specific enthalpy as state variables yields:

$$
\begin{aligned}
& V_{l} \cdot\left[\left(\frac{P}{\rho_{l}} \cdot\left(\frac{\partial \rho_{l}}{\partial P}\right)_{h}-1\right) \cdot \frac{d P}{d t}+\left(\frac{P}{\rho_{l}} \cdot\left(\frac{\partial \rho_{l}}{\partial h_{l}}\right)_{P}+\rho_{l}\right) \cdot \frac{d h_{l}}{d t}\right]= \\
& -\dot{m}_{l}^{o} \cdot\left(h_{l}^{o}-\left(h_{l}-\frac{P}{\rho_{l}}\right)\right)+\dot{m}_{\text {cond }} \cdot\left(h_{l}^{s a t}-\left(h_{l}-\frac{P}{\rho_{l}}\right)\right) \\
& +\left(1-x_{m v}\right) \cdot \dot{m}_{\text {drain }}^{e} \cdot\left(h_{\text {drain_l }}^{e}-\left(h_{1}-\frac{P}{\rho_{l}}\right)\right)+W_{v l}-W_{l w}
\end{aligned}
$$

$$
\begin{aligned}
& V_{v} \cdot\left[\left(\frac{P}{\rho_{v}} \cdot\left(\frac{\partial \rho_{v}}{\partial P}\right)_{h}-1\right) \cdot \frac{d P}{d t}+\left(\frac{P}{\rho_{v}} \cdot\left(\frac{\partial \rho_{v}}{\partial h_{v}}\right)_{P}+\rho_{v}\right) \cdot \frac{d h_{v}}{d t}\right]= \\
& \dot{m}_{v}^{e} \cdot\left(h_{v}^{e}-\left(h_{v}-\frac{P}{\rho_{v}}\right)\right)-\dot{m}_{\text {cond }} \cdot\left(h_{l}^{\text {sat }}-\left(h_{v}-\frac{P}{\rho_{v}}\right)\right) \\
& +x_{m v} \cdot \dot{m}_{\text {drain }}^{e} \cdot\left(h_{\text {drain }}^{e} v\right. \\
& \left.-\left(h_{v}-\frac{P}{\rho_{v}}\right)\right)-W_{v l}-W_{v w}-W_{1 t}
\end{aligned}
$$

Energy accumulation at the wall

$$
M_{w} \cdot c_{p w} \cdot \frac{d T_{w}}{d t}=W_{l w}+W_{v w}-W_{w a}
$$

Heat exchange between the liquid and steam phases

$$
W_{v l}=K_{v l} \cdot A_{v l} \cdot\left(T_{v}-T_{l}\right)
$$

Heat exchange between the liquid or steam phases and the wall

$$
\begin{aligned}
& W_{l w}=K_{l w} \cdot A_{l w} \cdot\left(T_{l}-T_{w}\right) \\
& W_{v w}=K_{v w} \cdot A_{v w} \cdot\left(T_{v}-T_{w}\right)
\end{aligned}
$$

Heat exchange between the condenser and the exter$\underline{\text { nal medium }}$

$$
W_{w a}=K_{w a} \cdot A_{w a} \cdot\left(T_{w}-T_{a}\right)
$$

Heat exchange between the liquid and the tube bundle 'Pipes'

$$
\begin{gathered}
\Delta W_{1}(i)=\beta \cdot h_{\text {conv }}(i) \cdot \Delta S_{\text {ext } 1} \cdot\left(T_{v}-T_{w 1}(i)\right)+W_{2 t} / N \\
W_{1 t}=\sum \Delta W_{1}(i)
\end{gathered}
$$

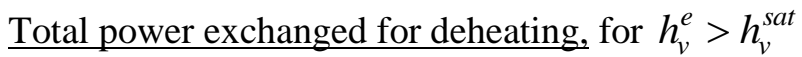

$$
W_{2 t}=\dot{m}_{v}^{e} \cdot\left(h_{v}^{e}-h_{v}^{s a t}\right)
$$

\section{$\underline{\text { Heat transfer convection coefficients }}$}

The Nusselt correlation is used to calculate the heat transfer coefficients $h_{\text {conv }}$ between the steam and the outside wall of the tube bundle, in the condensation zone.

The heat transfer coefficient between the liquid and the wall $\left(K_{l w}\right)$, the heat transfer coefficient between the steam and the wall $\left(K_{v w}\right)$ and the heat transfer coefficient between the steam and the liquid $\left(K_{v l}\right)$ were calculated for flat wall. 


\section{Test-case of the condenser}

\subsection{Modelica model of the condenser}

To simulate the complex dynamic physical behaviour in normal and dysfunctional conditions of the condenser model, a test model called "TestDynamicCondenser" has been developed by assembling the necessary components from the ThermoSysPro library (cf. Figure 6). The test model includes the level control system.

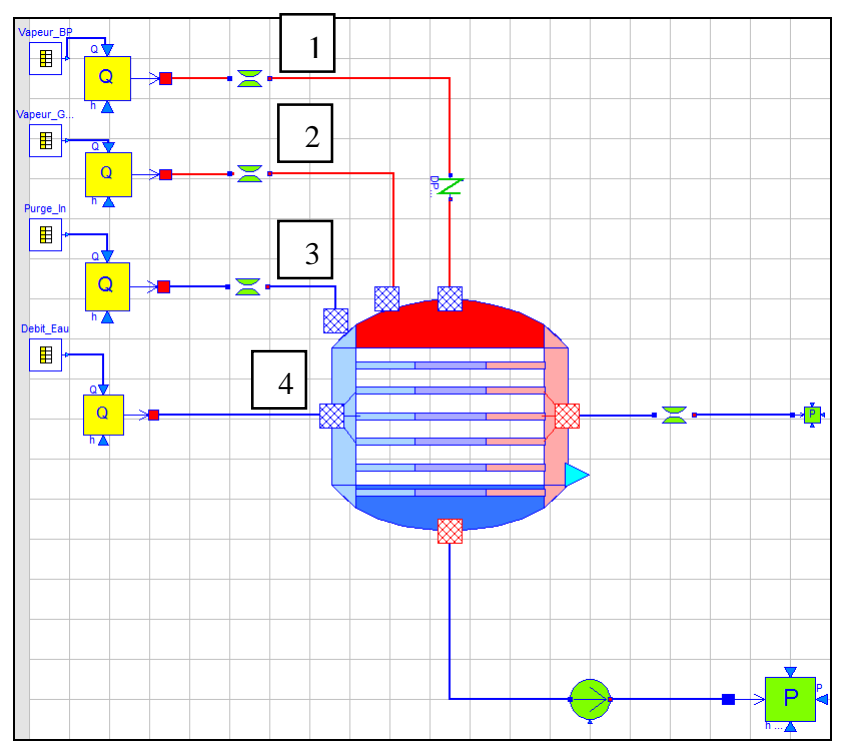

Figure 6: model of the condenser "TestDynamicCondenser"

\subsection{Data implemented in the model}

All geometrical data were provided to the model: tubes and exchangers lengths, diameters, volumes, corrective terms for the heat exchange coefficients, corrective terms for the pressure losses, etc. The plant characteristics are given in Figure 9 (cf. Appendix).

\subsection{Calibration of the model}

The calibration step consists in setting (blocking) the maximum number of thermodynamic variables to known measurement values. This method ensures that all needed performance parameters, size characteristics and output data can be computed.

For this model:
- setting (blocking) the cavity pressure to known measurement value and the value of corrective term of heat exchange coefficient between the cavity and pipes can be computed by model inversion,

- setting (blocking) the mass flow rate in the pump and the characteristics of the pump can be computed by model inversion (a polynomial coefficient)

\subsection{Simulation scenario}

In order to challenge the dynamic simulation capabilities of the model, a high amplitude transient "loss of the cold source pumps followed by a turbine trip and a missed islanding" is applied as a simulation scenario to the model. The purpose is to study the evolution of the pressure of the condenser for this type of transient. For this class of transients, the condenser pressure should always be less than $5 \mathrm{e} 4 \mathrm{~Pa}$ (maximum pressure for the availability of the condenser).

This transient is used to check and validate the physics taken into account in the model and the numerical robustness of the model as it runs the condenser model into very different operating regimes. This allows to assess the validity and applicability range of the model equations, and the numerical robustness of the Modelica implementation when using Dymola.

\subsection{Boundary conditions of the model}

The boundary conditions of the model (scenario profiles) are presented in Figure 7.

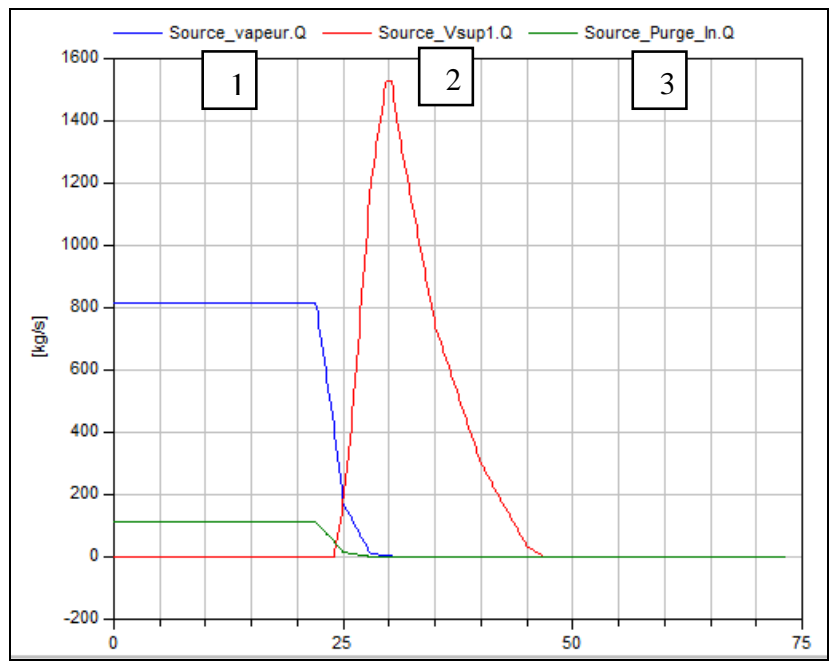

Figure 7a: input mass flow rate of the steam

Others data of the model:

\begin{tabular}{|l|l|l|}
\hline Enthalpy of the LP steam “1” & $2416.6 \mathrm{e} 3$ & $\mathrm{~J} / \mathrm{kg}$ \\
\hline Enthalpy of the HP steam “2” & $2759.6 \mathrm{e} 3$ & $\mathrm{~J} / \mathrm{kg}$ \\
\hline
\end{tabular}




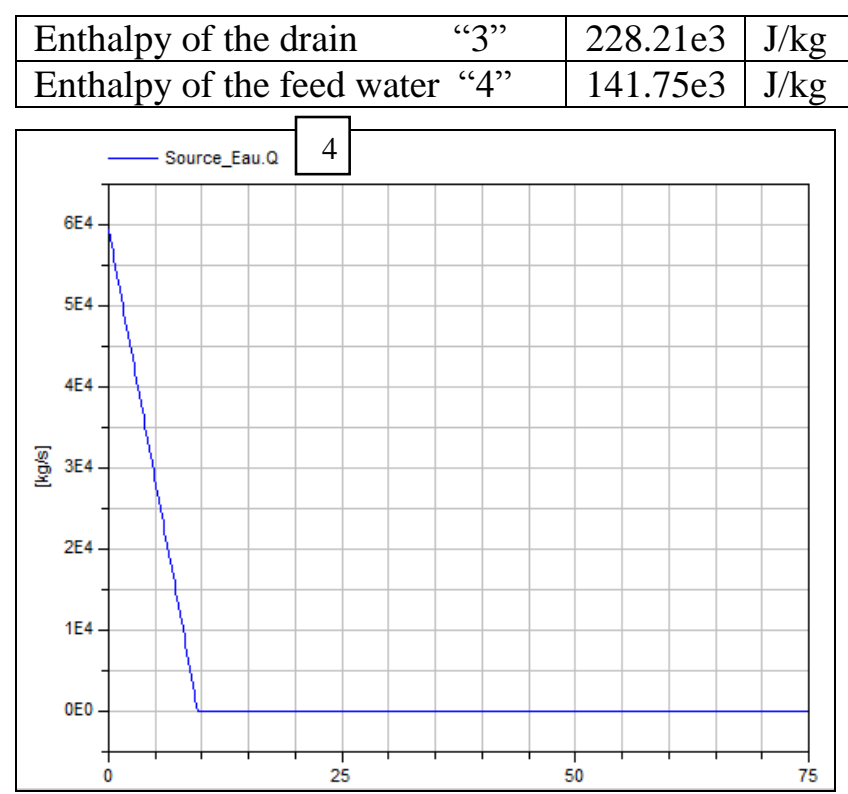

Figure 7b: input mass flow rate of the feedwater

\subsection{Results of dynamic simulations}

In order to cover the whole transient, the simulation time has been set at 75 seconds.

Simulation runs were done using Dymola 6.1 and Dymola 2012, with Dassl solver and with a tolerance $=0.0001$. The simulation of the scenarios were mostly successful, with only one iteration variable to be fed manually.

The following phenomena are simulated:

- local condensation,

- swell and shrink effect in cavity,

- cavity water levels.

The model is able to compute:

- the mass flow rate of the condensate,

- the thermal power of the condenser and tubes,

- the distribution of pressure, temperature and specific enthalpy inside the tube bundle,

- the cavity water level and cavity pressure.

The results of the simulation runs are given in Figure 8 . Figures $8 \mathrm{a}$ and $8 \mathrm{~b}$ show the results obtained with Dymola. The simulation result demonstrates that the maximum pressure in the condenser is less than $5 \mathrm{e} 4 \mathrm{~Pa}$ as required.

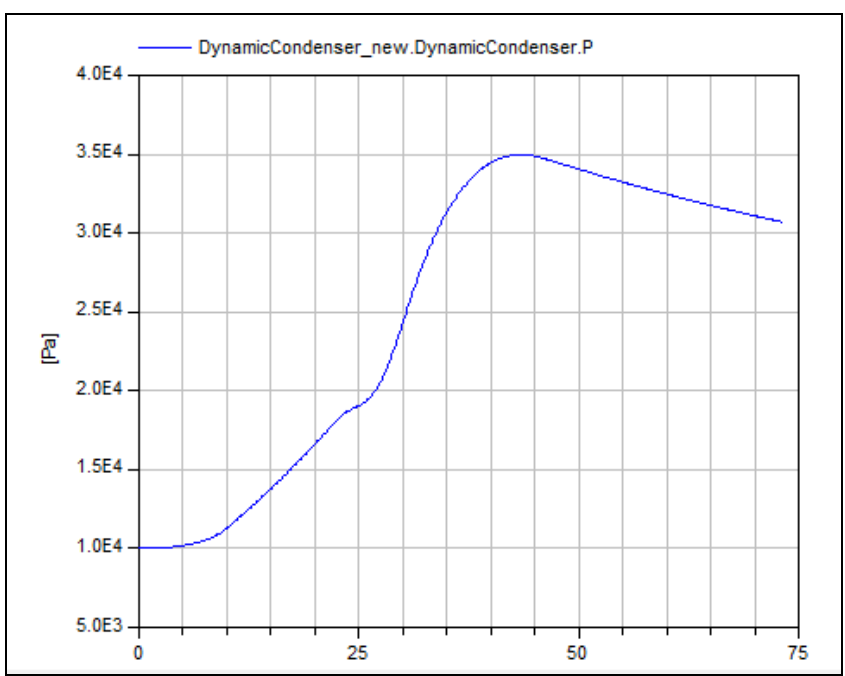

Figure 8a: evolution of the pressure inside the condenser

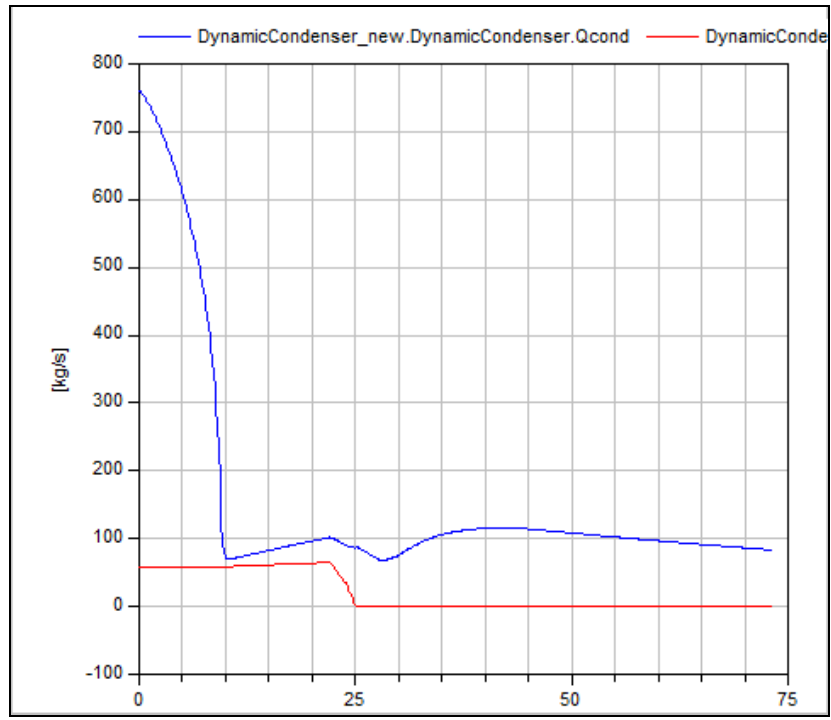

Figure 8b: evolution of the mass flow rate of the con-

$$
\text { densate }\left(--\dot{m}_{\text {cond_1 } 1},---\dot{m}_{\text {cond_2 } 2}\right)
$$

\section{Conclusion}

A new open source Modelica library called 'ThermoSysPro' has been developed within the framework of the ITEA 2 EUROSYSLIB project. This library has been mainly designed for the static and dynamic modeling of power plants, but can also be used for other energy systems such as industrial processes, buildings, etc. It is intended to be easily understood and extendable by the models developer.

A new dynamic model of a condenser has been developed using existing elements of ThermoSysPro. 
To validate the model, the following difficult transient was simulated: loss of cold source pumps followed by a turbine trip and a missed islanding.

The simulation result shows that the maximum pressure in the condenser is less than $5 \mathrm{e} 4 \mathrm{~Pa}$ (maximum pressure for the availability of the condenser) as required by the operation rules. The model enables to show that the evolution of the pressure inside the condenser strongly depends on the value of the condensation mass flow rate, the heat exchange between the vapor and the liquid at the bottom of the condenser and the thermal loss to the ambient.

\section{Nomenclature}

\begin{tabular}{|c|c|}
\hline \multicolumn{2}{|c|}{ Symbols } \\
\hline$\dot{m}$ & Mass flow \\
\hline$N$ & Number of segments for cooling pipes \\
\hline$\rho$ & Fluid density \\
\hline$h$ & Fluid specific enthalpy \\
\hline$u$ & Fluid specific internal energy \\
\hline$P$ & Fluid pressure \\
\hline$T$ & Fluid temperature \\
\hline$c_{p}$ & Fluid specific heat capacity \\
\hline$V$ & Volume \\
\hline$t$ & Time \\
\hline$W$ & Power \\
\hline$x_{v}$ & Vapor mass fraction in vapor phase \\
\hline$x_{l}$ & Vapor mass fraction in liquid phase \\
\hline$x_{m v}$ & Vapor mass fraction in input drain \\
\hline$z_{i}$ & Pipe inlet altitude \\
\hline$\Lambda$ & Friction coefficient \\
\hline$\zeta$ & Friction corrective coefficient \\
\hline$\beta$ & $\begin{array}{l}\text { Corrective term for heat exchange coef- } \\
\text { ficient }\end{array}$ \\
\hline$\Delta x$ & Tube segment length \\
\hline$\Delta S$ & Heat surface exchange of tube segment \\
\hline$D$ & Tube diameter \\
\hline$A$ & $\begin{array}{l}\text { Tube cross section or heat exchange } \\
\text { surface }\end{array}$ \\
\hline$e$ & Wall thickness \\
\hline$\lambda$ & Conduction coefficient \\
\hline$K$ & Heat exchange coefficient \\
\hline$M$ & Mass \\
\hline$h_{c}$ & Convective coefficient \\
\hline$h_{\text {conv }}$ & $\begin{array}{l}\text { Convective coefficient of heat transfer } \\
\text { by condensation between the vapor and }\end{array}$ \\
\hline
\end{tabular}

\begin{tabular}{|l|l|}
\hline & the tube bundle \\
\hline ntubes & Number of tubes in the bundle \\
\hline
\end{tabular}

Indices

\begin{tabular}{|l|l|}
\hline$X_{i}$ or $X(i)$ & Quantity in volume $i$ \\
\hline$X_{i: i+1}$ & $\begin{array}{l}\text { Flow of quantity between volume } i \\
\text { and volume } i+1\end{array}$ \\
\hline$X_{e}$ or $X^{e}$ & Quantity at inlet \\
\hline$X_{o}$ or $X^{o}$ & Quantity at outlet \\
\hline$X_{l}$ & Quantity relative to liquid \\
\hline$X_{v}$ & Quantity relative to vapor \\
\hline$X_{w}$ & Quantity relative to cavity wall \\
\hline$X_{w 1}$ & Quantity relative to pipes wall \\
\hline$X_{\text {ext }}$ & $\begin{array}{l}\text { Quantity relative to external side of } \\
\text { wall }\end{array}$ \\
\hline$X_{a}$ & Quantity relative to ambient \\
\hline$X^{\text {sat }}$ & Quantity relative to saturated phase \\
\hline$X_{\text {cond }}$ & Quantity relative to condensation \\
\hline$X_{\text {drain }}$ & Quantity relative to drain \\
\hline$X_{\text {drain_l }}$ & Quantity relative to drain, for liquid \\
\hline$X_{\text {drain_v }}$ & Quantity relative to drain, for vapor \\
\hline
\end{tabular}

\section{References}

[1] Bouskela D., Chip V., El Hefni B., Favennec J.M., Midou M. and Ninet J. 'New method to assess tube support plate clogging phenomena in steam generators of nuclear power plants', Mathematical and Computer Modelling of Dynamical Systems, 16: 3, 257-267, 2010.

[2] El Hefni B., Bouskela D., 'Modelling of a water/steam cycle of the combined cycle power plant "Rio Bravo 2" with Modelica', Modelica 2006 conference proceedings.

[3] David F., Souyri A., Marchais G., 'Modelling Steam Generators for Sodium Fast Reactors with Modelica', Modelica 2009 conference proceedings

[4] El Hefni B., Péchiné B., 'Model driven optimization of biomass CHP plant design', Mathmod conference 2009, Vienna, Austria.

El Hefni B., Bouskela D., 'Dynamic modelling of a combined cycle power 
plant with ThermoSysPro', Modelica 2011 conference proceedings

[6] Souyri A., Bouskela D., 'Pressurized Water Reactor Modelling with Modelica', Modelica 2006 conference proceedings.

[7] El Hefni B. 'Dynamic modeling of concentrated solar power plants with the ThermoSysPro Library' (Parabolic Trough collectors, Fresnel reflector and Solar-Hybrid, SolarPaces 2013, Elsevier's Energy Procedia.

[8] Baligh El Hefni, , Daniel Bouskela, Guillaume Gentilini 'Dynamic modelling of a water heater with the ThermoSysPro Library' Modelica2012

[9] Collier J.G., and Thome J.R., 'Convective Boiling and Condensation', $\mathrm{Mc}$ Graw-Hill Book Company (UK) limited, 1972 Clarendon Press, Oxford, 1996.

[10] Patankar S.V., 'Numerical Heat Transfer and Fluid Flow', Hemisphere Publishing Corporation, Taylor \& Francis, 1980. 


\section{Appendix}

\begin{tabular}{|c|c|c|c|}
\hline \multicolumn{4}{|l|}{ Component- } \\
\hline \multicolumn{4}{|c|}{ DynamicCondenser_new } \\
\hline \multicolumn{4}{|l|}{ Comment } \\
\hline \multicolumn{4}{|l|}{ Model- } \\
\hline \multicolumn{4}{|c|}{ ThermoSysPro.WaterSteam.HeatExchangers.DynamicCondenser } \\
\hline \multicolumn{4}{|c|}{ Comment Dynamic Cavity } \\
\hline \multicolumn{4}{|l|}{ Parameters- } \\
\hline Vo & $0.0463379>$ & & Fraction of initial liquid volume in the Cavity $[0<\mathrm{V} / 0<1]$ \\
\hline $\mathrm{P} 0 \mathrm{c}$ & $1 \mathrm{e} 4$, & $\mathrm{Pa}$ & INitial pressure in the Cavity \\
\hline Rv & $12.89873616>$ & m & Radius of the Cavity cross-sectional area \\
\hline Lv & 10.7903346 & m & Cavity length \\
\hline L2 & $15.4>$ & m & Pipes length \\
\hline Lc & $2.5>$ & m & support plate spacing in cooling zone(Chicanes] \\
\hline Dc & $0.018>$ & m & Internal diameter of the cooling pipes \\
\hline $\mathrm{ec}$ & $0.5 \mathrm{e}-3>$ & m & Thickness of the cooling pipes \\
\hline Ns & $5>$ & & Number of segments for pipes \\
\hline ntubest & $92916>$ & & Number of total pipes in Cavity \\
\hline ntubes $V$ & $220>$ & & Numbers of pipes in a vertical plan in Cavity \\
\hline $\mathrm{cp}$ & $573>$ & $\mathrm{J} /(\mathrm{kg} \cdot \mathrm{K})$ & Specific heat capacity of the metal of the cooling pipes \\
\hline tho & $4579>$ & $\mathrm{kg} / \mathrm{m} 3$ & Density of the metal of the cooling pipes \\
\hline lambda & \begin{tabular}{|r|} 
\\
\end{tabular} & $W /(m . K)$ & Wall thermal conductivity of the cooling pipes \\
\hline \multirow{2}{*}{$\begin{array}{l}\text { DynamicCondenser } \\
\text { pipe_3 }\end{array}$} & $=50 \mathrm{e}, \mathrm{z} \mid($ fixed=false, start=0.5), Kpa=0.01, Qcond(start=1000)] $\mathrm{E} \equiv$, & & \\
\hline & I, fixed=false]), advection=false, dynamic_mass_balance=false] $\equiv$ 三, & & \\
\hline $\mathrm{Ce} 2$ & ?[(h_vol(fixed=false, start=172390), h(fixed=false, start=172390)] $E$ 三 > & & Cooling water outlet \\
\hline
\end{tabular}

Figure 9: data of the model 